\title{
Introductory notes for the Acta IMEKO Special Issue on the XXIX Italian National Congress on Mechanical and Thermal Measurements
}

\author{
Alfredo Cigada1, Roberto Montanini² \\ ${ }^{1}$ Dipartimento di Meccanica., Politecnico di Milano, Via La Masa 1- 20156 Milano, Italy \\ 2 Dipartimento di Ingegneria, Università degli Studi di Messina, C.da di Dio - 98166 Villaggio Sant' Agata, Messina, Italy
}

\begin{abstract}
Section: EDITORIAL
Citation: Alfredo Cigada, Roberto Montanini, Introductory notes for the Acta IMEKO Special Issue on the XXIX Italian National Congress on Mechanical and Thermal Measurements, Acta IMEKO, vol. 10, no. 4, article 4, December 2021, identifier: IMEKO-ACTA-10 (2021)-04-04
\end{abstract}

Received December 14, 2021; In final form December 14, 2021; Published December 2021

Copyright: This is an open-access article distributed under the terms of the Creative Commons Attribution 3.0 License, which permits unrestricted use, distribution, and reproduction in any medium, provided the original author and source are credited.

Corresponding author: Alfredo Cigada, e-mail: alfredo.cigada@polimi.it

\section{Dear Readers,}

There is no doubt that Measurements are playing a fundamental role in our everyday life. Hot topics like the Internet of Things, Industry 4.0, Measurements for health or smart structures can not exist without a massive and pervasive presence of sensors and, more generally speaking, of Measurement Systems, meaning that data management and their analysis, both led by the interpretation by numerical models and by data driven approaches, are to be considered research trends of paramount interest for Measurements.

The reason why Mechanical Measurements are considered a science on its own has strong roots, not always fully understood, as data quality assessment, a preliminary step to any model, requires skills in a broad range of engineering topics, from sensors to mechanics, to materials and data science, just to mention some: this wide knowledge makes the cultural background of the expert in Mechanical Measurements one of the largest in Engineering.

All these aspects make the yearly Italian meeting of "Forum Nazionale delle Misure" a milestone for the discussion and update about the new trends in Measurements, a science running very fast: this is also the occasion to relate to the colleagues mainly dealing with Electric and Electronic Measurements, to widen the horizons and cultural exchange, making the occasion very rich for both a review on the present activities and a stimulus for the future.

The pandemic has created a discontinuity in the meeting long lasting tradition, started in 1986: in 2020 the Forum took place online, and the in-person meeting was organized again in September 2021 at Giardini Naxos, a wonderful location close to Messina.
As usual, part of the workshop has consisted in joint meetings between scientists from the Mechanical and Thermal Measurements and the Electrical and Electronic Measurements academic groups, in an interesting cultural discussion on the shared topics, faced with different viewpoints, and then on other topics, more peculiar to each group, in which the common language of Metrology has a fundamental role to define the measurements quality.

This special issue collects a selection of 12 papers presented during the three days of the "Forum": the authors have been asked to review their work and prepare an extended version, fit for the publication on ACTA IMEKO, a reference journal for Measurements.

The remarkable transversality of the Mechanical Measurements field is witnessed by the significant heterogeneity of the topics covered in each of the twelve selected works.

The paper entitled 'Skin potential response for stress recognition in simulated urban driving by Zontone et al. addresses the problem of stress conditions arising in car drivers using Machine Learning techniques based on Skin Potential Response (SPR) signals recorded from each hand of the test subjects. Results showed that, in a situation without traffic, the test individuals are less stressed, confirming the effectiveness of the proposed minimally invasive system for detection of stress in drivers.

In the paper 'Human identification and tracking using ultrawideband-vision data fusion in unstructured environments' the research group of the University of Trento, led by Prof. Mariolino De Cecco, faced the problem of the cooperation between Automated Guided Vehicles (AGV) and the operator, to solve two crucial functions of autonomy: operator identification, and tracking. Using sensor fusion, the authors were able to improve the accuracy and goodness of the final tracking, reducing uncertainty. 
The third paper, authored by Giulietti et al., deals with the continuous monitoring of cement-based structures and infrastructures, to optimize their service life and reduce maintenance costs. The proposed approach is based on electrical impedance measurements. Data can be made available on a Cloud through Wi-Fi network or LTE modem, hence they can be accessed remotely, via a user-friendly multi-platform interface.

The paper ' $V$ alidation of a measurement procedure for the assessment of the safety of buildings in urgent technical rescue operations' saw the collaboration between the research group of the University of L'Aquila and the Fire, Public Rescue and Civil Defense Department of the Italian Ministry of the Interior. The work provides a preliminary contribution to the draft of standard procedures for the adoption of Total Stations by rescuers in emergency situations, so as to offer a reliable and effective support to their assessment activities.

In the paper 'A comparison between aeroacoustic source mapping techniques for characterization of wind turbine blade models with microphone arrays', G. Battista et al. deal with the problem of characterizing the aeroacoustic noise sources generated by a rotating wind turbine blade, in order to provide useful information for tackling noise reduction. This paper discusses a series of acoustic mapping strategies that can be exploited in this kind of applications, based on laboratory tests carried out in a semianechoic room on a single-blade rotor.

The research group of the University of Padua, led by Prof. Stefano Debei, presented a paper entitled 'Occupancy grid mapping for rover navigation based on semantic segmentation', dealing with obstacle perception in a planetary environment by means of occupancy grid mapping. To evaluate the metrological performances of the proposed method, the ESA Katwijk Beach Planetary Rover Dataset have been used.

The paper, 'Characterization of glue behaviour under thermal and mechanical stress conditions' by Caposciutti et al., explores the behaviour of the glued interface commonly used to fix the electronics to the box housing, as it undergoes daily or seasonal thermal cycles combined to mechanical stress. To carry out the study, the Authors prepared some parallel plates capacitors by using glue as a dielectric material. Non-linear behaviour of the capacitance vs. temperature as well as effects of thermal cycles on the glue geometry were investigated.

The research group of the University of Perugia, led by Prof. Gianluca Rossi, presented an optical-flow-based motion compensation algorithm to be used in thermoelastic stress analysis to account for rigid-displacements that can occur during loading. The proposed approach is based on measuring the displacement field of the specimen directly from the thermal video. The blurring and edge effects produced by the motion were almost completely eliminated, making it possible to accurately measure the stress field, especially in areas around geometrical discontinuities.

Thermoelasticity and ArUco markers were also employed by L. Capponi et al. to validate a numerical model of the inspection Robot mounted on the new San Giorgio's Bridge on the Polcevera river in Genova. An infrared thermoelasticity-based approach was used to measure stress-concentration factors while ArUco fiducial markers were exploited to assess the natural frequencies of the Robot Inspection structure.

A completely different field of application concerns the paper 'Doppler flow phantom failure detection by combining empirical mode decomposition and independent component analysis with short time Fourier transform', that reports some very last results obtained by the research group led by Prof. Sciuto at the University of Roma Tre. The paper aims at providing an improvement of a previously proposed method for Doppler flow phantom failures detection, combining application of Empirical Mode Decomposition (EMD), Independent Component Analysis (ICA) and Short Time Fourier Transform (STFT) techniques on Pulsed Wave (PW) Doppler spectrograms.

The paper 'Comparison between 3D-reconstruction optical methods applied to bulge-tests through a feed-forward neural network' was originated from the collaboration between the research groups of Mechanical and Thermal Measurements of the University of Messina and of the University of Catania. The aim of the work was to compare two different $3 \mathrm{D}$ reconstruction techniques, epipolar geometry and Digital Image Correlation, to measure the deformation field of hyperelastic membranes under plane and equibiaxial stress state. A FFNN neural network was then used to assess accuracy of the two experimental approaches using a laser sensor as reference.

Finally, the paper 'Development and characterization of a self-powered measurement buoy prototype by means of piezoelectric energy harvester for monitoring activities in a marine environment', written by the research unit of the University of Messina, led by Prof. Roberto Montanini, addresses a series of interesting topics: among the others measurements for the sea and energy harvesting from the sea waves: the paper focuses on this aspect with an innovative approach.

We gratefully acknowledge all the Authors who have contributed to this special issue, as well as all the Reviewers and a special thanks goes to Prof. Francesco Lamonaca, Editor in Chief of ACTA IMEKO for his tireless and patient help which has made possible this special issue.

We are proud of having served as Guest Editors for this issue, hoping that this will help spreading the culture of Measurements.

Alfredo Cigada and Roberto Montanini

Guest Editors 